\title{
Dry needling could hold the key to relieving lower back pain
}

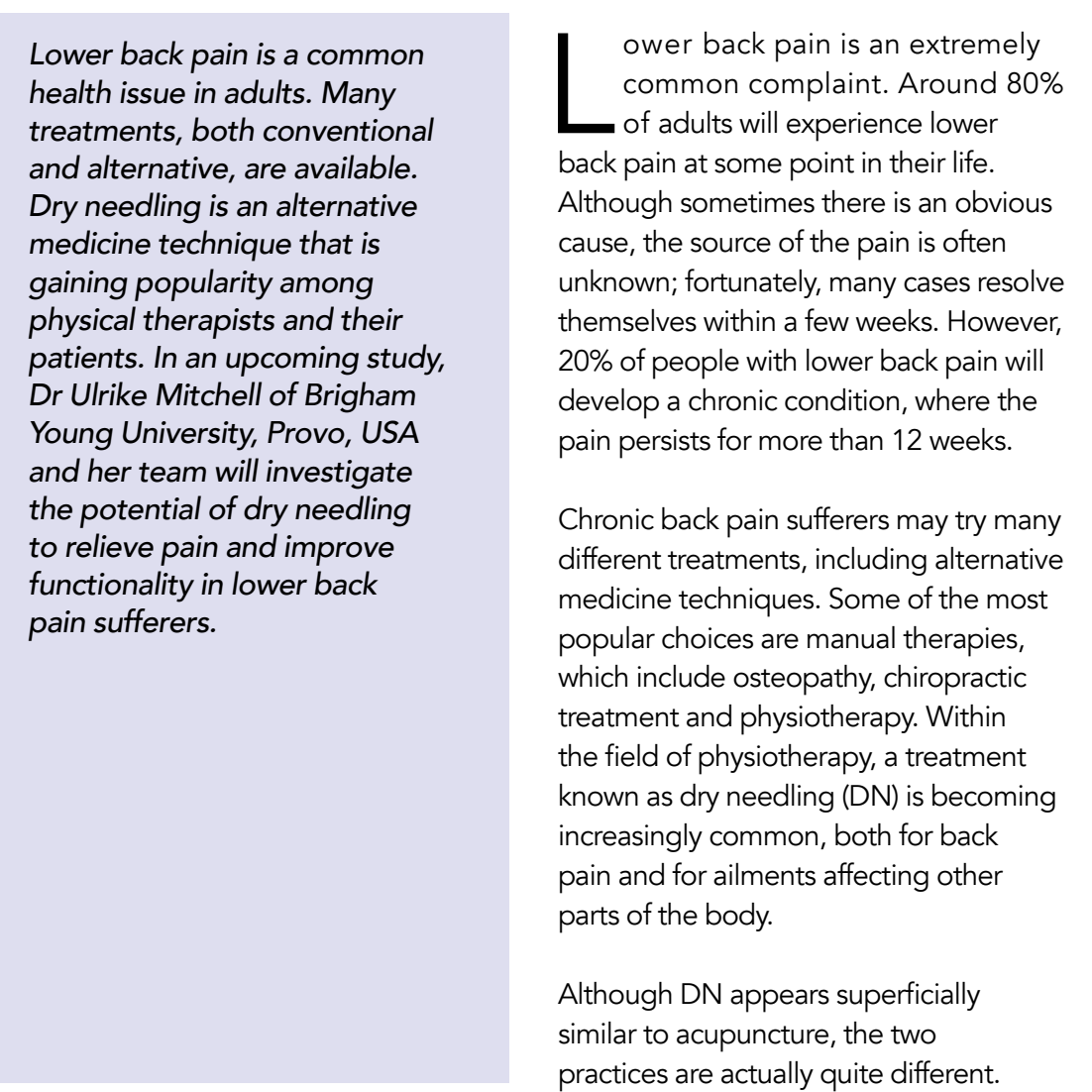

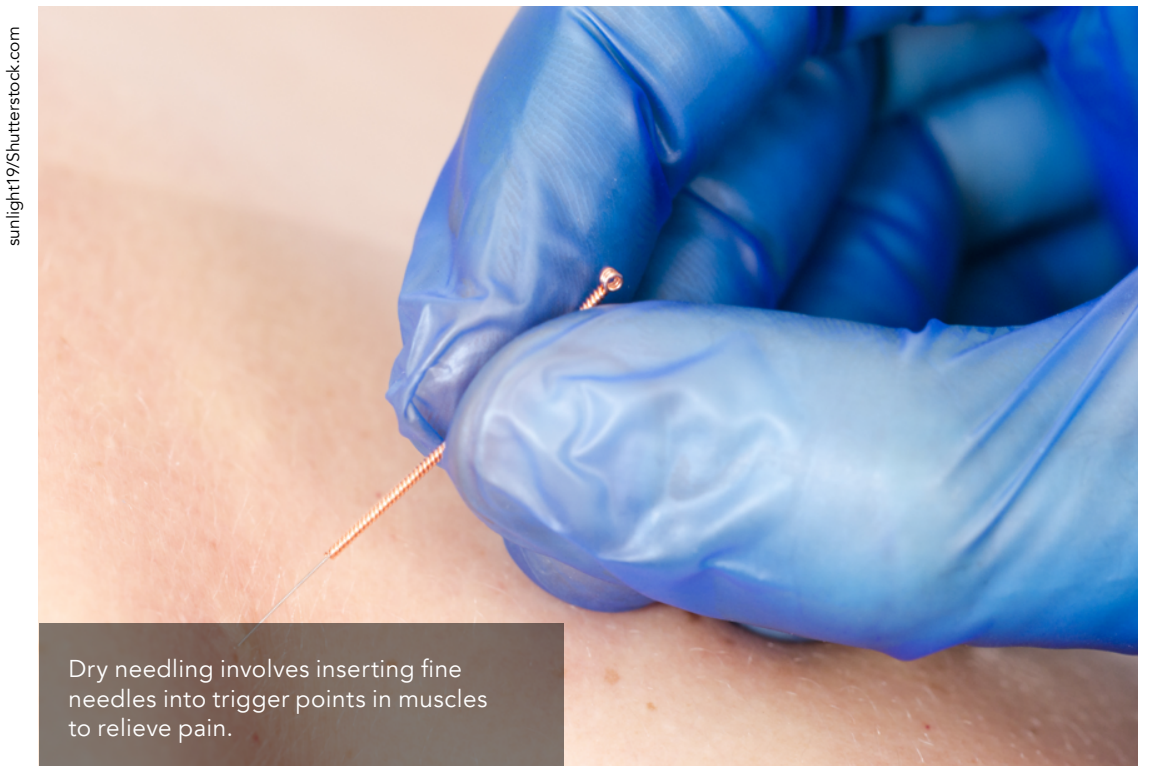

Acupuncture originates in traditional Chinese medicine and aims to treat ailments by altering the flow of "chi," or energy, within the body. In contrast practitioners of DN follow clear evidence-based guidelines to target specific problem areas, known as "trigge

During DN, a therapist uses very fine, needles. These are inserted into specific myofascial trigger points - which are sometimes called "muscle knots" underneath the skin. Trigger points are spots in muscles that produce pain when pressed or irritated. The best way to detect trigger points is a subject of debate but may largely rely on the skil and experience of the therapis.

\section{CAN DRY NEEDLING RELIEVE PAIN} AND IMPROVE MOVEMENT? The primary aim of $D N$ is to treat muscle pain and impaired movement. Exactly how this is achieved is currently poorly understood. However, it is needle is inserted into the trigger point, it creates a local muscle twitch, whic different theories have been suggested to explain this inactivation. Most of these involve the effect of DN on muscle fibres (individual muscle cells) and the nervous system, which controls muscle action

As with many alternative medicine techniques, the efficacy of $\mathrm{DN}$ is sometimes controversial. For example, one study has suggested no difference in treatment effect between DN and placebo, while others have shown that DN can effectively reduce pain when compared to other treatments. Other
studies have found that DN seems to

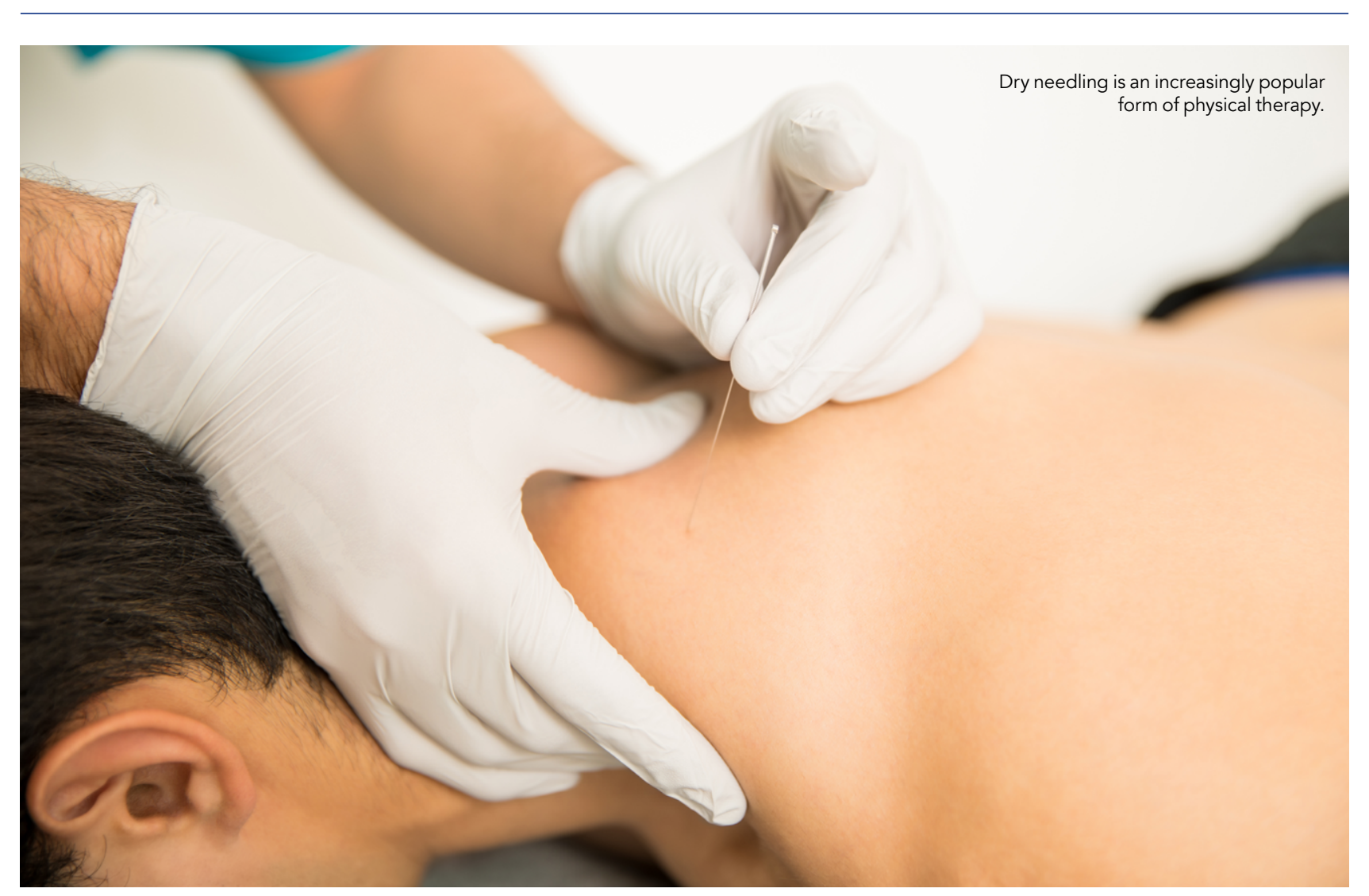

be better at reducing pain than either stretching or electric nerve stimulation, and at least as effective as manual trigger point release.

A NEW STUDY

Even though some evidence suggests that $\mathrm{DN}$ is effective in reducing pain,

a great deal remains unknown about how the technique works. Clearly, more research is needed if the full potential of $\mathrm{DN}$ is to be understood.

Dr Ulrike Mitchell and her team aim to fill some of the gaps in our knowledge on it as a method of pain relief. In a new study, Dr Mitchell will try to find the working mechanism behind $\mathrm{DN}$ while examining whether DN can improve functionality - in other words, reduce disability in back pain sufferers.

Previous studies have used DN in non-trigger point tissue as a control treatment. However, this may still have some physiological effects and is therefore not a true control treatme Instead, Dr Mitchell will use sham needles that she developed as a contro These are blunted needles that do not either DN or the sham -ns
Dry needling has been shown to be effective in reducing pain, but a great deal remains unknown about how it actually works.

In this first-of-its-kind study, Dr Mitchell and her team will investigate the effect of DNin three areas. pain, functionality

\section{DRY NEEDLING AND PAIN} defined as the minimum amount of pressure needed to cause the patient to feel pain. Previous studies have found that DN can increase the PPT, indicating a reduction in patient's pain. In this study Dr Mitchell will assess how the PPT changes after treatment in the two groups of patients: those who receive $\mathrm{DN}$, and the sham-needle treatment.

DRY NEEDLING AND FUNCTIONALITY ability, or functionality, due to lower DrMitche After one week of treatm

DRY NEEDLING: MECHANISM
Dr Mitchell will measure pain using the

Some patients suffer reduced physical functionality is higher in patients who have received DN, compared to those who Patients' functionality will be checked using several questionnaire-based assessments. One of these is the PatientSpecific Functional Scale, which is a particular treatment. Patients choose five putting their shoes on - and rate these on a 0-10 scale of difficulty. After treatment, patients rate the same activities on the change in functionality. Dr Mitchell hopes to discover whether DN of a particular trigger point can also increase the PPT of trigger points in other (a segment is part of the san segment (a segment is part of the spinal column,
made up of two vertebrae and the space patient's functionality before and after a important daily activities - for example, same scale, to see if there has been any 


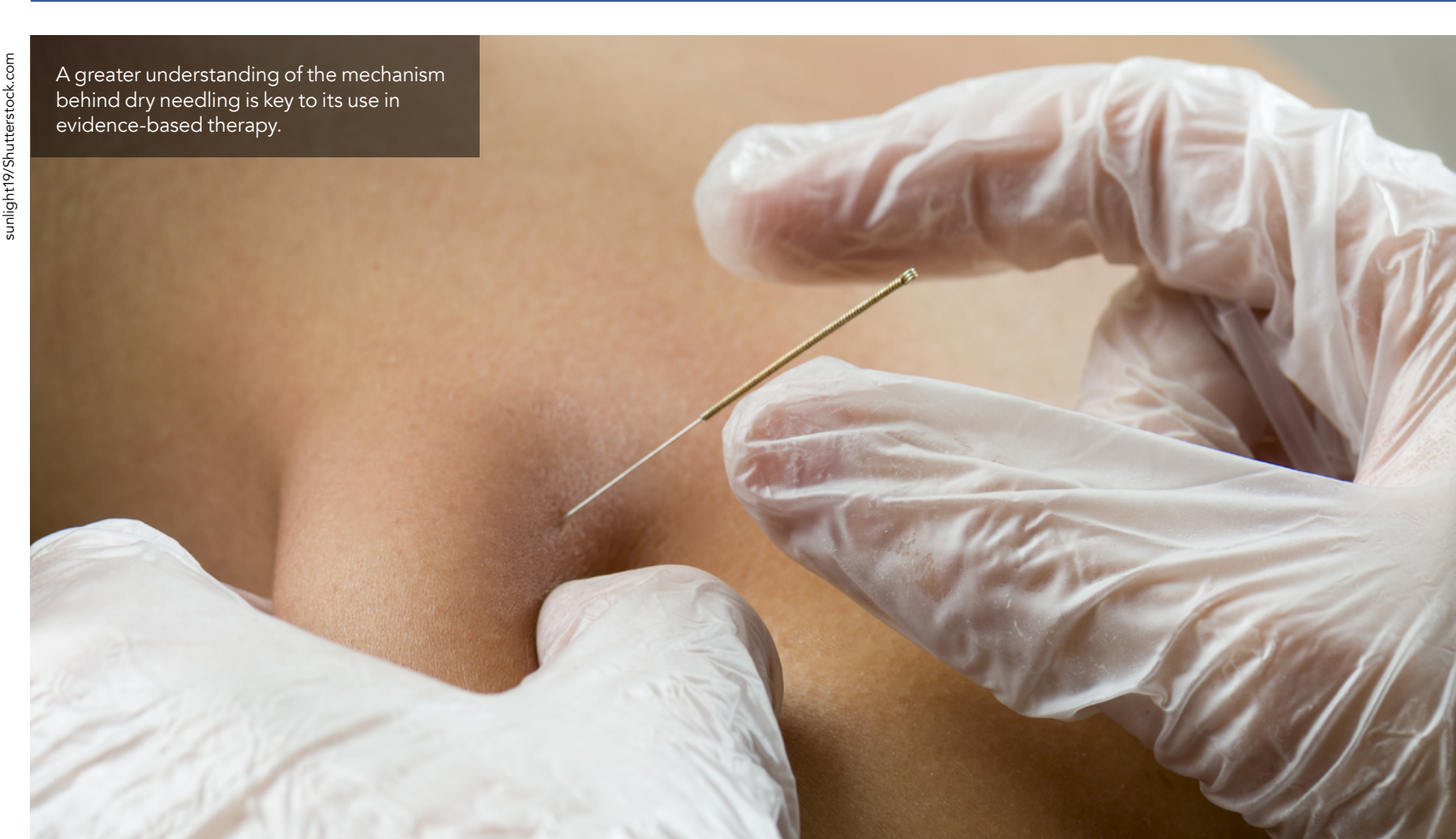

between them; in each segment, two assigned to the $\mathrm{DN}$ group, and half to the musclest, or even on the same side of the Both groups will receive two to throu. body. This will improve our knowledge "treatments" over the course of one of the mechanism by which $\mathrm{DN}$ can reduce pain.

Dr Mitchell and her team will recruit 50 patients, aged between 18 and 65 , to this study. These patients must have a painful trigger point in their lower alut gluteal muscles. Half will be week, as well as therapeutic exercises. Normally, a double-blind trial, where neither the physician/therapist nor the patient know whether they are in the treatment or control group, is ideal. In this case, it is impossible to blind the

It is essential that as techniques like dry needling increase in popularity, they are supported by a sound evidence base.

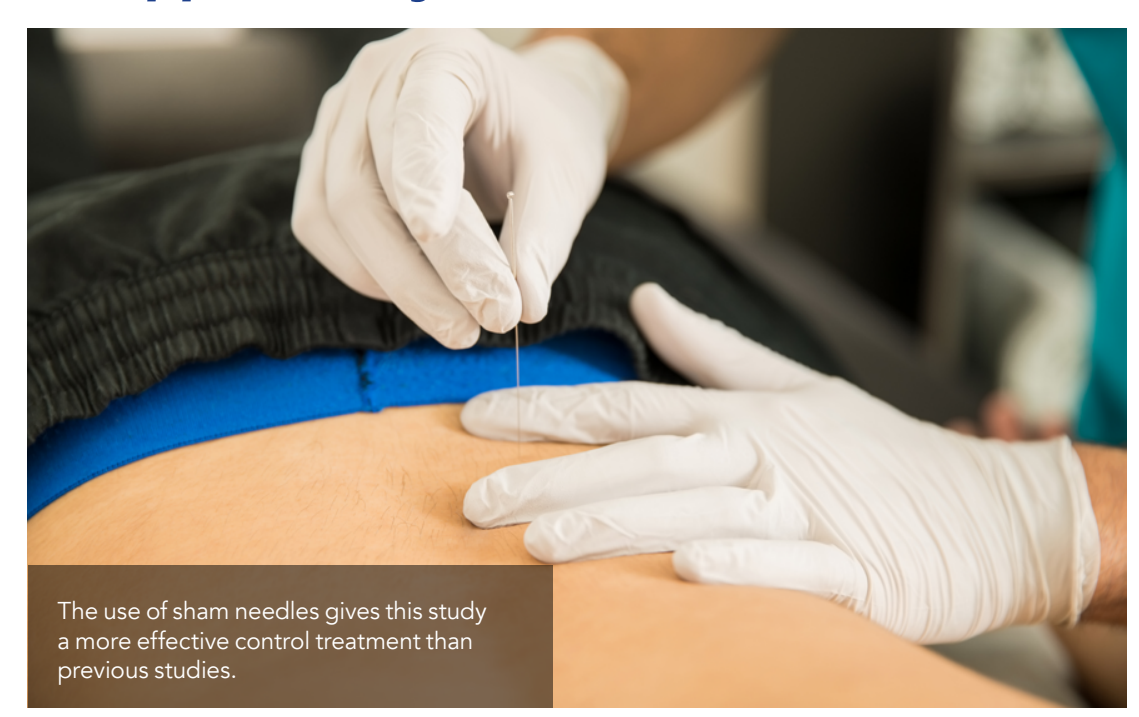

are using. Instead, to ensure a fair test, one therapist will perform both $\mathrm{DN}$ and sham-needle treatments, while another exercises

REVEALING THE FACTS Dr Mitchell and her team hypothesise that $D N$ will increase PPT in the lower back muscles, decrease lower treatment. In addition, DN of a spec trigger point should increase PPT in trigger points in other muscles innervated by the same segment (i.e. a control will give this study a leves of reliability that has previously been difficult to achieve.

Whatever the results, this study will add to the growing body of research around alternative medicine techniques therapy is an evidence-based skillset; it is therefore essential that as techniques such as DN increase in popularity, their use is supported by a sound evidence base. This allows both patients and benefits of their chosen treatment. If Dhsecomes a standard part of the physical therapist's toolkit, patients may be able to enjoy a smoother, quicker back pain and improve functionality, when compared to the sham-needle and muscle pain management. Physical their therapists to be confident in the

\section{Behind the Research}

Dr Ulrike Mitchell

E: rike@byu.edu T: +18014223344

\section{Research Objectives}

Dr Ulrike Mitchell aims to understand the working mechanism of dry needling.

\section{Detail}

Dr Ulrike Mitchell

Brigham Young University

Departis

Provo, UT 84602

USA

Bio

Ulrike Mitchell received her Bachelor of Physical Therapy in Germany, was manual therapy certified and received her advanced Master of Physical Therapy degree from the University of St. Augustine, FL, in 2000. She graduated from Brigham Young University, UT, in 2005 with a PhD in Exercise Science. She teaches at BYU Advanced Musculoskeletal Anatomy and Orthopaedic Impairment. Ulrike is an Orthopaedic Certified Specialist (OCS) and a Fellow of the American Academy of Orhopaedic Manual Physical Therapists. Her research is currently directed towards 1) the effects of dry needling and 2) the health

Funding

This project is in part funded by the American Academy of Orthopaedic Manual Physical Therapists (AAOMPT).

\section{Collaborators}

- Patrick Cook, PT (Baton Rouge, Louisiana) - Brad Pulsipher, PT (Salt Lake City, Utah) - Coleby Clawson, PT (Provo, Utah)

\section{References}

Mitchell, U. 2019. Does the addition of site-specific trigger point dry needling to physical therapy exercise intervention evoke segmental neuromodulation in the lower extremity and if so, do these changes

Mitchell, U et al. 2018. Positional changes in distance to the pleura and in muscle thickness for Dry Needling. to the pleura and
Physiotherapy

Mitchell, $U$ et al.2018. The construction of sham dry needles and their validity. Evidence-based Complementary and Alternative Medicine http://doi.org/10.1155/2018/956706

\section{Personal Response}

Do you think that dry needling has the potentia to replace other, more conventional physical therapy practices, or is it best used as just part

II I don't believe that DN will replace other practices, nor should it. It is an additional skill that physical however, only relatively few therapists take advant; of this opportunity. Those who do generally find DN to be very effective in treating muscular pain. DN should always only be used as an adjunct treatment, never as a stand-alone practice. DN is not the panacea for all based practice. The mystery surrounding its working mechanism makes it so interesting to research. 